\title{
GRAPH EQUATIONS FOR LINE GRAPHS, JUMP GRAPHS, MIDDLE GRAPHS, SPLITTING GRAPHS AND LINE SPLITTING GRAPHS
}

\section{B. Basavanagoud* and Veena Mathad**}

\begin{abstract}
For a graph $G$, let $\bar{G}, L(G), J(G) S(G), L(G)$ and $M(G)$ denote Complement, Line graph, Jump graph, Splitting graph, Line splifting graph and Middle graph respectively.

In this paper, we solve the graph equations $L(G)=S(H), M(G)=$ $S(H), L(G)=L_{s}(H), M(G)=L_{s}(H), J(G)=S(H), \overline{M(G)}=S(H), J(G)$ $=L_{s}(H)$ and $M(G)=L_{s}(G)$. The equality symbol ' $=$ ' stands for an isomorphism between two graphs.

Keywords: Line graph, Jump graph, Middle graph, Splitting graph, Line splitting graph.
\end{abstract}

* Dept. of Mathematics, Karnatak University, Dhanwad-580003. e-mail : bgouder1@yahoo.co.in

** Dept. of Mathematics, University of Mysore, Mysore-570006.e-mail : veena_mathad@rediffmail.com 


\section{Introduction}

By a graph, we mean a finite, undirected graph without loops or multiple edges. Definitions not given here may be found in [5]. For a graph $G, V(G)$ and $E(G)$ denote its vertex set and edge set respectively.

The open-neighborhood $N(u)$ of a vertex $u$ in $V(G)$ is the set of vertices adjacent to $u$ viz. $N(u)=\{v / u v \in E(G)\}$.

For each vertex $u_{i}$ of $G$, a new vertex $u_{i}^{\prime}$ is taken and the resulting set of vertices is denoted by $V_{1}(G)$.

The splitting graph $S(G)$ of a graph $G$ is defined as the graph having vertex set $V(G) \cup V_{1}(G)$ and two vertices are adjacent if they correspond to adjacent vertices of $G$ or one corresponds to a vertex $u_{i}^{\prime}$ of $V_{1}(G)$ and the other to a vertex $w_{i}$ of $G$ and $w_{i}$ is in $N\left(u_{i}\right)$. This concept was introduced by Sampathkumar and Walikar in [7].

The open-neighborhood $N\left(e_{i}\right)$ of an edge $e_{i}$ in $E(G)$ is the set of edges adjacent to $e_{i}$ viz. $N\left(e_{i}\right)=\left\{e_{i} / e_{i}\right.$ and $e_{i}$ are adjacent in $\left.G\right\}$.

For each edge $e_{i}$ of $G$, a new vertex $e_{i} \varangle$ is taken and the resulting set of vertices is denoted by $E_{1}(G)$.

The line splitting graph $L_{s}(G)$ of a graph $G$ is defined as the graph having vertex set $E(G) \cup E_{1}(G)$ with two vertices adjacent if they correspond to adjacent edges of $G$ or one corresponds to an element $e_{i}^{\prime}$ of $E_{1}(G)$ and the other to an elemente of $E(G)$ where $e_{i}$ is in $N\left(e_{i}\right)$. This concept was introduced by Kulli and Biradar in [6].

The jump graph $J(G)$ of $G$ is the graph whose vertices are edges of $G$ and two vertices of $J(G)$ are adjacent if and only if they are not adjacent in $G$. Equivalently, the jump graph $J(G)$ of $G$ is the complement of the line graph $L(G)$ of $G$. This concept was introduced by Chartrand in [3].

The middle graph $M(G)$ of a graph $G$ is the graph whose vertex set is $V(G) \cup E(G)$ and two vertices are adjacent if they are adjacent edges of $G$ or one is a vertex and other is an edge incident with it. This concept was introduced by Akiyama, Hamada and Yoshimura in [1]. 
In this paper, we solve the following graph equations:
I. $\quad L(G)=S(H)$
V. $J(G)=S(H)$
II. $\quad M(G)=S(H)$
VI. $\overline{M(G)}=S(H)$
III. $L(G)=L_{s}(H)$
Vil. $J(G)=L_{s}(H)$
IV. $M(G)=L_{s}(H)$
VIII. $\overline{M(G)}=L_{s}(H)$

Beineke has shown in [2] that a graph $G$ is a line graph if and only if $G$ has none of the nine specified graphs $F_{i} i=1,2, \ldots, 9$ as an induced subgraph. We depict here three of the nine graphs which we use. They are $F_{1}=K_{1,3^{\prime}} F_{3}$ (see Figure l (a)), and $F_{5}$ [see Figure $1(b)$ ]

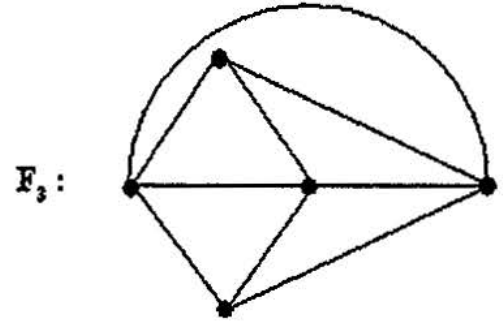

(a)

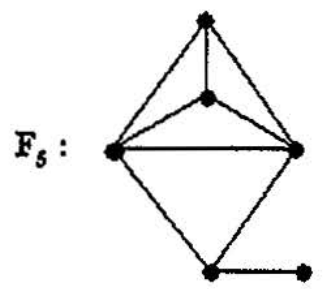

(b)

Figure 1

A graph $G^{+}$is the endedge graph of a graph $G$ if $G^{+}$is obtained from $G$ by adjoining an endedge $u_{i} u_{i} \notin$ at each vertex $u_{i}$ of $G$. Hamada and Yoshimura have proved in [4] that $M(G)=L\left(G^{+}\right)$.

\section{The Solution of $L(G)=S(H)$}

Any graph $\mathrm{H}$ which is a solution of the above equation, satisfies the following properties:

i) $\mathrm{H}$ must be a line graph, since $H$ is an induced subgraph of $S(H)$.

ii) $H$ does not contain a cut-vertex, since otherwise, $F_{1}$ is an induced subgraph of $S(H)$. 
iii) $H$ does not contain a vertex which is adjacent to two nonadjacent vertices, since otherwise, $F_{1}$ is an induced subgraph of $S(H)$.

iv) $H$ does not contain $C_{n^{\prime}} n \geq 4$ as a subgraph since otherwise, $F_{1}$ is an induced subgraph of $S(H)$.

It follows from above observations that $H$ has no cut-vertices. We consider the following cases:

Case 1. Suppose $H$ is disconnected. Then components of $H$ are $K_{1}$ or $K_{2}$ or $K_{3}$. Therefore, $\left(2 n K_{2} n K_{1}\right), n \geq 2 ;\left(n P_{5^{\prime}} n K_{2}\right), n \geq 2 ;\left(n K_{3}^{+}, n K_{3}\right), n \geq 2 ;\left(2 m K_{2} \cup n P_{5}\right.$, $\left.m K_{1} \cup n K_{2}\right), m, n \geq 1 ;\left(2 m K_{2} \cup n K_{3}^{+}, m K_{1} \cup n K_{3}\right), m, n \geq 1 ;\left(m P_{5} \cup n K_{3}^{+}, m K_{2} \cup\right.$ $\left.n K_{3}\right), m, n \geq 1$; and $\left(2 m K_{2} \cup n P_{5} \cup K_{3}^{+}, m K_{1} \cup n K_{2} \cup K_{3}\right), m, n, 1 \geq 1$ are the solutions.

Case 2. Suppose $H$ is connected. Then $H$ is $K_{1}$ or $K_{2}$ or $K_{3}$. The corresponding $G$ is $2 K_{2}$ or $P_{5}$ or $K_{3}^{+}$respectively.

From the above discussion, we conclude the following :

Theorem 1. The following pairs $(G, H)$ are all pairs of graphs satisfying the graph equation $L(G)=S(H)$ :

$\left(n P_{5}, n K_{2}\right), n \geq 1 ;\left(2 n K_{2}, n K_{1}\right), n \geq 1 ;\left(n K_{3}^{+}, n K_{3}\right), n \geq 1 ;\left(2 m K_{2} \cup n P_{5}, m K_{1} \cup\right.$ $\left.n K_{2}\right), m, n \geq 1 ;\left(2 m K_{2} \cup n K_{3}^{+}, m K_{1} \cup n K_{3}\right), m, n \geq 1 ;\left(m P_{5} \cup, m K_{2} \cup n K_{3}\right), m, n$ $\geq 1 ;$ and $\left(2 m K_{2} \cup n P_{5} \cup, K_{3}^{+}, m K_{1} n K_{2} \cup\left(K_{3}\right), m, n, I \geq 1\right.$.

\section{The solution of $M(G)=S(H)$}

We have investigated the solutions $(G, H)$ of equation 2 in Theorem 1. Among these solutions $\left(2 n K_{2}, n K_{1}\right), n \geq 1 ;\left(n K_{3}^{+}, n K_{3}\right), n \geq 1 ;$ and $\left(2 m K_{2} \cup n K_{3}^{+}, m K_{1} \cup\right.$ $\left.n K_{3}\right), m, n \geq 1$ are as $\left(G^{+}, H\right)$. Therefore, the solutions of the equation 3 are $\left(2 n K_{1}\right.$, $\left.n K_{1}\right) n \geq 1,\left(n K_{3}, n K_{3}\right), n \geq 1$ and $\left(2 m K_{1} \cup n K_{3}, m K_{1} \cup n K_{3}\right), m, n \geq 1$. Thus we have the following.

Theorem 2. The solutions $(G, H)$ of the graph equation $M(G)=S(H)$ are $\left(2 n K_{1}\right.$, $\left.n K_{1}\right) n \geq 1 ;\left(n K_{3}, n K_{3}\right), n \geq 1 ;$ and $\left(2 m K_{1} \cup n K_{3}, m K_{1} \cup n K_{3}\right), m, n \geq 1$. 


\section{The Solution of $L(G)=L_{s}(H)$}

We observe that in this case $\mathrm{H}$ satisfies the following properties:

i) $\mathrm{H}$ does not contain a component having more than one cut-vertex since otherwise, $F_{1}$ is an induced subgraph of $L_{s}(H)$.

ii) $H$ is not a complete graph $K_{n^{\prime}} n \geq 4$, since otherwise, $F_{1}$ is an induced subgraph of $L_{s}(H)$.

iii) $H$ does not contain $P_{4}$ as a subgraph since otherwise, $F_{1}$ is an induced subgraph of $L_{s}(H)$.

iv) $H$ does not contain $K_{1,4}$ as an induced subgraph since othenwise, $F_{1}$ is an induced subgraph of $L_{s}(H)$.

v) $H$ is not a cycle $C_{n^{\prime}} n \geq 4$ since otherwise, $F$, is an induced subgraph of $L_{s}(H)$.

vi) $H$ does not contain a cut-vertex which lies on blocks other than $K_{2}$.

From observation (i) it follows that every component of $\mathrm{H}$ has at most one cutvertex. We consider the following cases.

Case 1. Suppose $H$ has no cut-vertices. Then $H$ is $n K_{2}, n \geq 1$ or $n K_{3}, n \geq 1$, or $m K_{2} \cup n K_{3}, m, n \geq 1$.

For $H=n K_{2}, n \geq 1, \quad G=2 n K_{2}$

For $H=n K_{3}, n \geq 1, \quad G=n K_{3}^{+}$

For $H=m K_{2} \cup n K_{3}, m, n \geq 1, G=2 m K_{2} \cup n K_{3}^{+}$

Case 2. Suppose $H$ has cut-vertices. We consider the following subcases :

Subcase 2.1. Assume $H$ is connected. Then $H$ is $K_{1,2}$ or $K_{1,3}$. The corresponding $G$ is $P_{5}$ or $n K_{3}^{+}$respectively.

Subcase 2.2. Assume $H$ is disconnected. Then each component of. $H$ has atmost one cut-vertex. Then $H$ is $m K_{1,2} \cup n K_{1,3} \cup K_{2} \cup r K_{3}, m \geq 1$ and $n, l, r \geq 0$ or $m K_{1,2}$ $\cup n K_{1,3} \cup \mathbb{K}_{2} \cup r K_{3}, n \geq l$ and $m, l, r \geq 0$. In this case $\left(m P_{5} \cup(n+r) K_{3}^{+} \cup 2 m K_{2}\right.$, $m K_{1,2} \cup n K_{1,3} \cup\left(K_{2} \cup r K_{3}\right)$ is the solution. 
From above discussions, we conclude the following:

Theorem 3. The following pairs $(G, H)$ are all pairs of graphs satisfying the graph equation $L(G)=L_{s}(H)$ :

$\left(2 n K_{2}, n K_{2}\right), n \geq 1 ;\left(n K_{3}^{+}, n K_{3}\right), n \geq 1 ;\left(2 m K_{2} \cup n K_{3}^{+}, m K_{2} \cup n K_{3}\right), m, n \geq 1 ;\left(m P_{5}\right.$ $\left.\cup(n+r) K_{3}^{+} \cup 2 K_{2^{\prime}}, m K_{1,2} \cup n K_{1,3} \cup \mathbb{K}_{2} \cup r K_{3}\right), m \geq 1, n, l, r \geq 0$; and $\left(m P_{5} \cup\right.$ $\left.(n+r) K_{3}^{+} \cup 2 I K_{2}, m K_{1,2} \cup n K_{1,3} \cup I K_{2} \cup r K_{3}\right), n \geq 7, m, l, r \geq 0$.

\section{The Solution of $M(G)=L_{s}(H)$}

Theorem 3 provides solutions of the equation $L(G)=L_{s}(H)$. Among these solutions $\left(2 n K_{2}, n K_{2}\right), n \geq 1 ;\left(n K_{3}^{+}, n K_{3}\right), n \geq 1 ;\left(2 m K_{2} \cup n K_{3}^{+}, m K_{2} \cup n K_{3}\right), m, n \geq 1 ;$ and $\left(m P_{5} \cup(n+r) n K_{3}^{+} \cup 2 I K_{2}, m K_{1,2} \cup n K_{1,3} \cup I K_{2} \cup r K_{3}\right), r, l \geq 0, n \geq l$ and $m=0$ are as $\left(G^{+}, H\right)$. Therefore, solutions of equation 5 are $\left(2 n K_{1}, n K_{2}\right), n \geq 1 ;\left(n K_{3}, n K_{3}\right), n$ $\geq 1 ;\left(2 m K_{1} \cup n K_{3}, m K_{2} \cup n K_{3}\right), m, n \geq 1$; and $\left((n+r) K_{3} \cup 2 / K_{1}, n K_{1,3} \cup\left(K_{2} \cup r K_{3}\right)\right.$, $n \geq 1$ and $r, l \geq 0$. Now we state the following result.

Theorem 4. The solutions $(G, H)$ of the graph equation $M(G)=L_{s}(H)$ are $\left(2 n K_{1}\right.$, $\left.n K_{2}\right), n \geq 1 ;\left(n K_{3}, n K_{3}\right), n \geq 1 ;\left(2 m K_{1} \cup n K_{3}, m K_{2} \cup n K_{3}\right), m, n \geq 1 ;$ and $\left\langle(n+r) K_{3}\right.$ $\left.\cup 2 K_{1}, n K_{1,3} \cup \mathbb{K}_{2} \cup r K_{3}\right), n \geq 1$ and $r, 1 \geq 0$.

\section{The Solution of $J(G)=S(H)$}

First, we observe that in this case $\mathrm{H}$ satisfies the following properties:

i) If $H$ has atleast one edge, then it is connected since otherwise, $\bar{F}_{3}$ is an induced subgraph of $S(H)$.

ii) $H$ does not contain a cut-vertex, since otherwise, $\bar{F}_{3}$ is an induced subgraph of $S(H)$.

iii) If $H$ is a block, then it is a complete graph since otherwise, $\bar{F}_{5}$ is an induced subgraph of $S(H)$.

It follows from observations (i), (ii) and (iii), that $H$ is $n K_{1}, n \geq 1$ or $K_{n^{\prime}} n \geq 2$. The corresponding $G$ is $K_{1,2 n}$ or, $K_{1, n}^{+}-v$ where $v$ is a pendant vertex of $K_{1, n}^{+}$which is adjacent to the vertex of maximum degree respectively. 
Hence equation 6 is solved and solutions are given in the following theorem.

Theorem 5. The following pairs $(G, H)$ are all pairs of graphs satisfying the graph equation $J(G)=S(H)$ :

$\left(K_{1,2 n^{\prime}} n K_{1}\right) n \geq 1$; and $\left(K_{i, n}^{+}, K_{n}\right) n \geq 2$ where $v$ is a pendant vertex of which is adjacent to the vertex of maximum degree.

\section{The Solution of $\overline{M(G)}=S(H)$}

Theorem 5 gives solutions for the equation $J(G)=S(H)$. But none of these is as $\left(G^{+}, H\right)$. Hence, there is no solution of the equation $\overline{M(G)}=S(H)$.

Thus we have the following result.

Theorem 6. There is no solution of the graph equation $\overline{M(G)}=S(H)$.

\section{The Solution of $J(G)=L_{s}(H)$}

In this case $\mathrm{H}$ satisfies the following properties:

i) $H$ does not contain more than one cut-vertex, since otherwise, $\bar{F}_{5}$ is an induced subgraph of $L_{s}(H)$.

ii) $H$ does not contain a cut-vertex, which lies on blocks otherthan $K_{2}$, since otherwise, $\bar{F}_{5}$ is an induced subgraph of $L_{s}(H)$.

iii) $H$ does not contain a cycle $C_{n^{\prime}} n \geq 4$, since otherwise, $\bar{F}_{5}$ is an induced subgraph of $L_{s}(H)$.

iv) If $\mathrm{H}$ is disconnected graph then every component of $\mathrm{H}$ is $\mathrm{K}_{2^{\prime}}$, since otherwise, $\bar{F}_{3}$ is an induced subgraph of $L_{s}(H)$.

We consider the following cases.

Case 1. Suppose $H$ is disconnected. Then from observation (iv), $H$ is $n K_{2}, n \geq 2$. The corresponding $G$ is $2 n K_{2}$. 
Case 2. Suppose $H$ is connected. Then from observation (i), $H$ has atmost one cut-vertex. We consider the following subcases.

Subcase 2.1. Assume $H$ has a cut-vertex. Then from observation (ii), $H$ is $K_{1, n^{\prime}} n$ $\geq 2$. The corresponding $G$ is $K_{l, n}^{+}-v$, where $v$ is a pendant vertex adjacent to the vertex of maximum degree.

Subcase 2.2. Assume $H$ is a block. Then $H$ is $K_{2}$ or $K_{3}$. The corresponding $G$ is $K_{1,2}$ or $K_{1,3}^{+}$, where $v$ is a pendant vertex of $K_{1,3}^{+}$adiacent to a vertex of maximum degree.

Thus equation 8 is solved and we have the following.

Theorem 7. The following pairs $(G, H)$ are all pairs of graphs satisfying the graph equation $J(G)=L_{s}(H)$ :

$\left(2 n K_{2}, n K_{2}\right), n \geq 2 ;\left(K_{1,2}, K_{2}\right) ;\left(K_{1,3}^{+}-v, K_{3}\right)$, where $v$ is a pendant vertex adjacent to the vertex of maximum degree; and $\left(K_{i, n}^{+}-v, K_{1, n}\right), n \geq 2$, where $v$ is a pendant vertex adjacent to the vertex of maximum degree.

\section{The Solution of $\overline{M(G)}=L_{s}(H)$}

Theorem 7 gives solutions for the equation $J(G)=L_{s}(H)$. Among these $\left(2 n K_{2}, n K_{2}\right)$, $n \geq 2$ is of the form $\left(G^{+}, H\right)$. Therefore, the solution of the equation is $\left(2 n K_{1}, n K_{2}\right)$, $\overline{M(G)}=L_{s}(H)$ is $\left(2 n K_{1}, n K_{2}\right), n \geq 2$.

Now, we state the following result.

Theorem 8. The solutions of the graph equation are $\overline{M(G)}=L_{s}(H)$ are $\left(2 n K_{1}\right.$, $\left.n K_{2}\right), n \geq 2$. 


\section{References}

1. J.Akiyama, T.Hamada and I.Yoshimura, (1974), Miscellaneous properties of middle grophs, TRU Mathematics, 10, 41-53.

2. L.W.Beineke, (1967), On derived graphs and digraphs, in : Beitröge zur Graphentheorie (Monebach 1967), 17-24.

3. G.T.Chartrand, H. Hevia, E.B.Jarette, and M.Schulty, (1997), Subgraph distance in graphs defined by edge transfers, Discrefe Mathematics, 170, 63-79.

4. T.Hamada and I.Yoshimura, (1976), Traversability and Connectivity of the middle graph of a graph, Discrete Mathematics, 14, 247-256.

5. F.Harary, (1969), Graph Theory, Addison-Wesley, Reading, Mass,.

6. V.R.Kulli and M.S.Biradar, (2002), The line-splitting graph of graph, Acta Ciencia Indica, Vol. XXVIII M.No. 3, 317-322.

7. E.Sampathkumar and H.B.Walikar, (1980-81), On splitting graph of a graph, J. Karnotak Univ. Sci., 25 and 26 (combined), 13-16. 